\title{
Functions of MAPK Cascade Pathways in Plant Defense Signaling
}

\author{
Yong Hwa Cheong ${ }^{1 *}$ and Min Chul Kim ${ }^{2}$ \\ ${ }^{1}$ Department of Bio-Environmental Science, Sunchon National University, Suncheon 540-742, Korea \\ ${ }^{2}$ Department of Agronomy and Division of Applied Life Science, Gyeongsang National University, Jinju 660-701, Korea \\ (Received on January 15, 2010; Accepted on April 8, 2010)
}

\begin{abstract}
Protein phosphorylation is one of the major mechanisms for controlling many cellular processes in all living organisms. Mitogen-activated protein kinase (MAPK) cascades are known to transducer extracellular stimuli to several cellular processes, including cell division, differentiation as well as responses to various stresses. In plants, several studies have revealed that MAPK cascade pathways play an important role in responses against biotic and abiotic stresses, including wounding, pathogen infection, temperature, drought, salinity and plant hormones. It is also known that MAPK cascadesmediated signaling is an essential process in the resistance step to pathogens by regulating the activity of transcription factors. Here, the insights into the functions of MAPK cascade pathways in plant defense response signaling from Arabidopsis, tobacco and rice are described.
\end{abstract}

Keywords : defense signaling, MAPK pathways, pathogen infection, transcription factor

Plants have developed complex signaling and defense systems to protect themselves from the attack of pathogen by hypersensitive response (HR)-cell death at the site of pathogen invasion, the generation of several signaling molecules, such as reactive oxygen species (ROS), salicylic acid (SA), jasmonic acid (JA), ethylene, and NO (nitric oxide), induction of pathogenesis-related (PR) gene expression, and the accumulation of antimicrobial compounds (Dangl and Jones, 2001).

MAPK cascades are well known as highly conserved signaling molecules found in all eukaryotes, including animals, fungi and plants. These MAPK cascade pathways consists of three interacting proteins including MAPKs, MAPK kinases (MAPKK/MKKs) and MAPKK kinases (MAPKKK/MEKKs) by linking upstream receptors to downstream targets (Hamel et al., 2006). In a typical MAPK cascade pathways, MAPKs are activated by MAPKKs via dual phosphorylation of conserved threonine and tyrosine residues in the TxY motif, which is located in the activation

\footnotetext{
*Corresponding author.

Phone) +82-61-750-3298, FAX) +82-61-752-8011

E-mail) yhcheong@sunchon.ac.kr
}

loop (T-loop) between kinase subdomains VII and VIII. MAPKKs are themselves activated by MAPKKKs through phosphorylation of conserved serine/threonine residues in the S/T-X3-5-S/T motif, also located in the T-loop (Jonak et al., 2002). In general, MAPKKKs are activated by physically interacting with a receptor and becoming phosphorylated either by the receptor itself or an interlinking upstream protein kinase (Chang and Karin, 2001). Among the modules of MAPK cascades, mitogen-activated protein kinases (MAPKs) are well known as serine/threoninespecific kinases that play important roles in many signal transduction pathways involved in cell growth, differentiation, and stress response (Chang and Karin, 2001).

In plants, MAPK cascade pathways are also known to be associated with various cellular processes including the regulation of development, growth, programmed cell death and responses to a variety of environmental stimuli, such as pathogen infection, wounding, low temperature, drought, high salinity, touch and reactive oxygen species (Colcombet and Hirt, 2008; Pitzschke et al., 2009). MAPK cascade pathways have been found in large number throughout a variety of plant species, including Arabidopsis, tobacco, tomato, alfalfa and rice (Agrawal et al., 2003; Colcombet and Hirt, 2008; Hamel et al., 2006; MAPK group, 2002). In the Arabidopsis genome, 20 MAPKs, 10 MAPKKs and 80 MAPKKKs have been identified (MAPK group, 2002). Although a large of genes associated in MAPK cascades have been identified based on the sequence analysis of genome, a few MAPK cascades have been determined in plant defense signaling (Pitzschke et al., 2009). Recent several studies have been reported to identity the direct MAPK transcriptional targets and the insights into the mechanism by which MAPK signaling networks regulate gene expression in plant by activation of transcriptional factors (Fiil et al., 2009). In this review, the roles of MAPK cascades pathways involved in plant defense signaling against infections from Arabidopsis, tobacco and rice are described.

\section{MAPK families involved in plant defense pathways}

Although the serine/threonine kinase domain are highly conserved in all plant MAPKs (Hanks et al., 1988), it has 
been identified that some plant MAPKs have additional Nterminal or C-terminal domains, which show greater sequence divergence than the kinase catalytic domain. Based on the phylogenic analysis with their overall deduced amino acid sequences, plant MAPKs can be divided into four sub-family groups (I-IV) (Agrawal et al., 2003; Hamel et al., 2006; MAPK group, 2002). Group I-III MAPKs possess a TEY motif in their activation loop cluster, whereas group IV MAPKs all display a distinctive TDY motif rather than TEY motif (Hamel et al., 2006).

Group I MAPKs are well known to be involved in a variety of environmental and hormonal responses (Colcombet and Hirt, 2008; Hamel et al., 2006). These groups include Arabidopsis AtMPK3 and AtMPK6 which activated by pathogen infection, osmotic stress and oxidative stress (Asai et al., 2002; Colcombet and Hirt, 2008; Ichimura et al., 2000), tobacco SIPK and WIPK which activated by salicylic acid (SA) or wounding (Zhang and Klessig, 2001), alfalfa SIMK and SAMK which involved in both biotic and abiotic stress responses (Zwerger and Hirt, 2001). Especially in wounding signal transduction, tobacco WIPK activated a transcription factor NtWIF, which involved in activation expression of a number of wound and pathogen response genes (Seo et al., 1995; Yap et al., 2005). Group II MAPKs are also well known to be involved in environmental stress responses, although these are less well-studied than the group I MAPKs. These groups include the Arabidopsis MPK4, which involved in plant defense signaling. The Arabidopsis mpk4 mutant displays a constitutive systemic acquired resistance (SAR) phenotype against pathogen infection (Petersen et al., 2000). The level of MPK4 protein is also activated with both biotic- and abiotic-stress treatments, such as low temperature, high salt and wounding (Ichimura et al., 2000). Group III MAPKs is very little known in plant stress signal pathways, although these groups also contain a TEY motif in their T-loop like groups I and II MAPKs. So far, only MPK7 which known as group III have shown to be regulated in a circadianrhythm-dependent manner (Schaffer et al., 2001). Group IV MAPKs possess a TDY motif instead of TEY motif in their T-loop, as well as an extended C-terminal domain. These group IV MAPKs include Arabidopsis AtMPK8, AtMPK9 and AtMPK15, BWMK1 from rice and TDY1 from alfalfa. Several studies suggested that OsBWMK1 and TDY1 are induced by blast fungus and wounding, respectively (Cheong et al., 2003; He et al., 1999; Schoenbeck et al., 1999). It have been well studied that OsBWMK1 plays an important role in rice defense signaling by activation of transcription factors, such as OsEREBP and OsWRKY33 (Cheong et al., 2003; Koo et al., 2009). Interestingly, the rice genome contains many more MAPKs with the TDY motif (11 genes) than with TEY motif (6 genes). However, Arabidopsis genome contain more MAPKs with TEY motif (12 genes) than with the TDY motif (8 genes) (Reyna and Yang, 2006).

\section{MAPKK (MKK) families involved in plant defense pathways}

So far, twenty-one MAPKK (MKK) genes have been identified in plants including Arabidopsis MKK1-5, alfalfa SIMKK and PRKK, tobacco NtMEK1-2 and SIPKK, tomato LeMEK1, and maize ZmMEK1 (Hamel et al., 2006; MAPK group, 2002). Plant MAPKKs have the motif $\mathrm{S} / \mathrm{T}-\mathrm{X}_{5}-\mathrm{S} / \mathrm{T}$ as a phosphorylation site which differs from their mammalian counterparts. Plant MAPKKs also contains a putative N-terminal MAPK-docking domain that is characterized by a cluster of basic and hydrophobic residues $\left(\mathrm{K} / \mathrm{R}-\mathrm{K} / \mathrm{R}-\mathrm{K} / \mathrm{R}-\mathrm{X}_{1-6}-\mathrm{LX}-\mathrm{L} / \mathrm{V} / \mathrm{I}\right)$. In the Arabidopsis genome, 10 MAPKK genes are identified and can be classified into four different groups (A-D) (MAPK group, 2002). So far, group A-C MAPKK genes are indentified in plant stress response pathways.

The group A MAPKKs include Arabidopsis MKK1 and MKK2, which well known to be upstream regulators for MPK4 and activated by wounding and abiotic stress (Meszaros et al., 2006). Arabidopsis MKK2 also acts as an upstream activator of MPK6 in addition to MPK4, and is known to mediate cold and salt stress signaling in plants (Teige et al., 2004). MKK2 is activated by MEKK1 which is known as the stress-inducible MAPKKK. MKK2 overexpressing plants exhibited constitutive MPK4 and MPK6 kinase activity, constitutively induction stress-induced marker gene expression, and increased freezing and salt tolerance. In contrast, $m k k 2$ null mutant plants showed hypersensitive to salt and cold stress (Teige et al., 2004). The group B MAPKKs have an unusual structural feature having a nuclear transport factor 2 (NTF2) domain in their extended C-terminus region and include Arabidopsis MKK3 and tobacco NPK2 (Quimby et al., 2000). The group C MAPKKs contain the alfalfa SIMKK which activate SIMK and mediate both salt and elicitor-induced signal pathways (Kiegerl et al., 2000). These groups also contain another tobacco NtMEK2, which is involved in not only activation of the SIPK and WIPK but also hypersensitive cell death in leaves (Yang et al., 2001).

\section{MAPKKK (MEKK) gene families involved in plant defense pathways}

The MAPKKK (MEKK) family forms the largest group components of MAPK cascade pathway found in plants. Among 80 putative MAPKKK genes identified in Arabidopsis genome, plant MAPKKKs can be divided into two 
subfamilies, including the MEKK-like protein kinases that are most similar to animal MEKKKs and Raf-like protein kinases (MAPK group, 2002). MEKK-like protein kinases include alfalfa OMTK1 (oxidative stress-activated MAP triplekinase 1), Arabidopsis AtMEKK1, and tobacco NPK1 (Mizoguchi et al., 1996; Nakagami et al., 2004; Nishihama et al., 2001). Members of the plant Raf-like protein kinases include EDR1 (enhanced disease resistance 1) and CTR1 (constitutive triple response 1) from Arabidopsis (Frye et al., 2001; Kieber et al., 1993). It have also been reported that AtMEKK1 acts as an upstream activator of MKK1-2, and MKK4-5 in plant defense response against pathogen as well as in abiotic stress conditions (Asai et al., 2002; Ichimura et al., 2006; Tiege et al., 2004).

It is also known that EDR1, a Raf-like MAPKKK, plays functions as a negative regulator in plant defense resistance into pathogen. Mutant edrl lines confer resistance to Erysiphe cichoracearum, a fungus that causes powdery mildew disease. Furthermore, kinase-deficient EDR1 overexpressing plants showed the enhanced resistance to powdery mildew (Frye et al., 2001; Tang and Innes, 2002). The CTR1, another Raf-like MAPKKK, acts as a negative regulator in ethylene signaling (Kieber et al., 1993). Mutant ctr1 plants display a constitutive triple response in the absence of ethylene, suggesting that the ethylene receptors ETR1 and ETR2 constitutively activate CTR1 in the absence of ethylene (Kieber et al., 1993).

\section{Arabidopsis MAPK cascade pathways in plant defense signaling}

A number of plant MAPK-related genes have been identified to be involved in the defense response against pathogens (Asai et al., 2002; Colcombet and Hirt, 2008; Pitzschke et al., 2009). Of the 20 identified MAPKs in Arabidopsis, only three MAPKs such as MPK3, MPK4 and MPK6 are known as key regulators in plant defense signaling and are activated by bacterial and fungal PAMPs (pathogen-associated molecular patterns). On the basis of amino acid sequence, MPK3 and MPK6 are closely related proteins and show a high level of functional redundancy. Both MAPKs are key regulators of a several cellular processes including defense response to bacterial and fungal pathogens (Colcombet and Hirt, 2008; Pitzschke et al., 2009). Several studies suggested that the MAPK cascade module MEKK1-MKK4/MKK5-MPK3/MPK6 is a critical signaling pathway linking the flagellin (flg22) receptor FLS2/BAK1 to the activation of the downstream transcription factor WRKY22 and WRKY29 genes. This MAPK cascade allows the early flg22-induced expression of WRKY29 and FRK1 gene. Transient overexpression of constitutively active form of MEKK1 showed the resistance to bacterial and fungal pathogens in leaves by activating several downstream events. Expression of constitutively active forms of MKK4, MKK5, and WRKY29 also showed the similar results in Arabidopsis (Asai et al., 2002). The MPK6 is also known to be involved specifically in fungal defense. MPK6-silenced Arabidopsis plants displayed resistance to fungal pathogen, Peronospora parasitica and to bacterial pathogen, Pseudomonas syringae strain (Menke et al., 2004).

Several studies have been demonstrated on downstream target protein of flg22-mediated MPK3/MPK6 pathways (Colcombet and Hirt, 2008; Pitzschke et al., 2009). Upon flg22 treatment, MPK6 specially phosphorylates the ACS6 (ACC synthase 6) which known as an enzyme involved in ethylene biosynthesis (Liu and Zhang, 2004). Recently, VIP1, a bZIP transcription factor, have been shown to be phosphorylated by MPK3 and in turn re-localized from the cytoplasm to the nucleus for activating PR-genes (Djamei et al., 2007).

It have been also reported that MPK3/MPK6 are important regulators in camalexin and ethylene biosynthesis. In plants, several fungal pathogens trigger the camalexin biosynthesis which known as a major phytoalexin compound. Ren et al. (2008) demonstrated that mpk3 and mpk6 mutant plants showed the reduction of camelexin production and more susceptible to fungal pathogen. In contrast, the activation of MPK3/MPK6 in MKK4/MKK5- or MEKK1-overexpressing plants is sufficient to induce the camalexin accumulation, showing that MPK3/MPK6 play a key role in camalexin-mediated pathogen resistance. Furthermore, the MKK9-MPK3/MPK6 module is also involved in ethylene biosynthesis during defense signaling responses (Xu et al., 2008).

Another MAPK cascades MEKK1-MKK1/2-MPK4 pathway have been also identified in plant defense signaling. Several papers reported that AtMPK4 acts as a regulator of pathogen defense responses, by demonstrating that MPK4 negatively regulate the accumulation of salicylic acid (SA), but positively regulate the JA-dependent signaling pathway (Petersen et al., 2000). mpk4 mutant plants exhibited enhanced resistance pathogens with activation of downstream effects, including elevation of SA levels, the activation of systemic acquired resistance, and constitutive expression of PR- genes. However, mpk4 mutant plants didn't show much changed in the induction of JA-responsive genes, PDF1.2 and THI2.1, upon methyl jasmonate treatment, suggesting that MPK4 are also required for JA-mediated gene expression during defense signaling (Petersen et al., 2000).

It was also recently reported that MPK4 regulates SAdependent and JA/ethylene (ET)-dependent responses via regulating the EDS1 and PAD4 (Brodersen et al., 2006). mpk4 mutant plants were shown to be defective in induc- 
tion of ethylene-responsive defense genes as well as being more susceptible to pathogen Alternaria brassicicola. Mutations of EDS1 and PAD4 abolished the de-repression of the SA pathway and suppressed the inhibition of the JA/ ET pathway in mpk4 mutants, indicating that MPK4 negatively regulates both EDS1 and PAD4 (Brodersen et al., 2006). Several studies have been reported to unveil the downstream target of MEKK1-MKK1-MPK4 pathway in plant defense signaling. The WRKY53 transcription factor may be partially responsible in mekklmutant, demonstrating that MEKK1 directly interacts with WRKY53 and alters the activity of transcription factor (Miao et al., 2007). So far, three proteins have been identified as downstream target protein of MPK4 including WRKY33, WRKY25 and MKS1 (Andreasson et al., 2005). MKS1 (MAP kinase substrate 1) was shown to couple MPK4 to the WRKY transcription factors WRKY25 and WRKY33. MKS1 overexpressing plants showed enhancement of the activation of SA-dependent resistance such as PR1 gene expression, SA accumulation, dwarfism and resistance to pathogen, but did not seem to affect the induction of JA-related defense genes (Andreasson et al., 2005). A recent report demonstrated that the transcription factors WRKY25 and WRKY33 interact with MKS1 in yeast, suggesting that these two WRKYs regulate the gene expression downstream of MPK4-mediated signaling (Qiu et al., 2008). Analysis of T-DNA mutants indicated that MEKK1 is required for flg22-induced activation of MPK4 but not MPK3 or MPK6 (Colcombet and Hirt, 2008; Meszaros et al., 2006; Pitzschke et al., 2009). MPK4 activity was increased within a few minutes with flg22 treatments and this activation is abolished in mekk1 mutant plant.

Interestingly, mekkl mutant lines showed that the kinase activity of MEKK1 may not be required for flg-22-induced MPK4 activation or for other macroscopic FLS2-mediated responses and displayed a severe dwarf phenotype, constitutive callose deposition and constitutive expression of pathogen response genes, indicating MEKK1 acts upstream of MPK4 as a negative regulator in plant defense signaling (Ichimura et al., 2006; Suarez-Rodriguez et al., 2007).

\section{Tobacco MAPK cascade pathways in plant defense signaling}

It was well known that the first key MAPK to be identified in plant defense signaling was the tobacco salicylic acid (SA)-induced protein kinase, SIPK (Zhang and Klessig, 1997). SIPK and another wound-induced protein kinase, WIPK have been demonstrated to be activated in response to many biotic and abiotic stresses (Zhang and Klessig, 1998; Zhang and Liu, 2001; Zhang et al., 2000). In defense signaling, tobacco SIPK and WIPK, which are homologue of Arabidopsis MPK6 and MPK3, respectively, are activated both by general elicitors and by the interaction of racespecific Avr protein and their cognate resistance proteins. R-gene, Cf-9-overexpressing transgenic tobacco plants exhibited the induction of WIPK and SIPK activity upon Avr9, fungal avirulence protein treatment (Zhang and Klessig, 1998). SIPK and WIPK are also activated in an $\mathrm{N}$ resistance gene-mediated manner upon infection with TMV (tobacco mosaic virus), in turn resulting in HR-like cell death in tobacco (Zhang and Klessig, 1998; Zhang and Liu, 2001; Zhang et al., 2000). Transient expression of SIPK by activation with NtMEK2 upstream kinase has been shown to be sufficient to activate defense-related gene expression and HR-like cell death (Yang et al., 2001). It have been reported that the tobacco NtMEK2-SIPK/WIPK pathway also plays an important role in $\mathrm{N}$ gene-mediated resistance (Jin et al., 2003). The induction of SIPK and WIPK activity enhanced the gene expression of HMGR which known as a key enzyme in the phytoalexin biosynthesis pathway (Zhang and Liu, 2001).

Several papers have been reported to identify the downstream target protein of NtMEK2-SIPK/WIPK pathway in tobacco. NtWIF, a transcription factor which involved in wound and pathogen responsive gene expression, might be activated by WIPK (Yap et al., 2005). Kim and Zhang (2004) also reported that the NtMEK2-SIPK/WIPK cascade can activate members of the WRKY family transcription factors which involved in transcription of disease resistance genes. Moreover, Menke et al. (2005) reported that SIPK can phosphorylate and activate WRKY1 and co-expression of WRKY1 and SIPK showed enhanced cell death than overexpression of either alone. Constitutively active NtMEK $^{\mathrm{DD}}$ overexpressing transgenic tobacco upregulated the Spm-induced HR related marker genes including HIN1, HMGR and HSR2003J (Takahashi et al., 2004) and elicited the HR-like cell death which is transduced by the activation of SIPK and WIPK (Yang et al., 2001). These results suggest that the NtMEK2-SIPK/WIPK module is one of key regulators in tobacco defense signaling and controls multiple defense responses to pathogen invasion. Furthermore, loss-of-function of virus-induced gene silencing (VIGS) demonstrated that NtMEK2, SIPK and WIPK are required for resistance to TMV by regulating the activity of certain downstream transcription factors, such as WRKYs and MYBs (Jin et al., 2003).

\section{Rice MAPK cascade pathways in plant defense sig- naling}

In Arabidopsis and tobacco, MAPK cascade pathways in defense signaling have been well established as mentioned earlier. However, little is known about the function of rice 
MAPK gene family in defense signaling so far. In rice genome, 17 MAPK gene family was identified through an in silico search (Reyna and Yang, 2006). Among them, only six MAPKs, including OsWJUMK1, OsMAPK4, OsBWMK1, OsMAPK6, OsMAPK 5 and OsBIMK2, have been characterized in rice (Agrawal et al., 2003; Cheong et al., 2003; Fu et al., 2002; He et al., 1999; Lieberherr et al., 2005; Song et al., 2006; Xiong and Yang, 2003). Of these six, OsBWMK1, OsMAPK5, OsMAPK6 and OsBIMK2 genes have been identified as a MAPK regulator in defense responses. Rice OsMAPK5 (also known as OsMSRMK2, OsMAPK2, OsMAP1 or OsBIMK1), an ortholog of Arabidopsis MPK3, was shown to be activated by various biotic and abiotic stresses as well as by abscisic acid (Xiong and Yang, 2003). RNAi-suppression lines of OsMAPK5 displayed constitutive PR gene expression and enhanced resistance to fungal and bacterial infection. In addition, overexpression of OsMAPK5 also showed tolerance to drought, salt and cold stress, suggesting that OsMAPK5 acts as a positive regulator of abiotic stress tolerance but acts as a negative regulator of rice disease resistance (Xiong and Yang, 2003).

OsMAPK6 is also known as an important regulator in rice disease resistance. Suppressing OsMAPK6 or knocking out of OsMAPK6 showed the enhanced rice resistance against bacterial pathogen Xanthomonas oryzae which causes the bacterial blight disease in worldwide. The resistant rice plants showed increased expression of several defense-responsive genes which involved in the NH1 (an Arabidopsis NPR1 orthologue)-mediated defense signal transduction pathway (Yuan et al., 2007). It have been also reported that a novel rice MAPK gene, OsBIMK2 (Oryzae sativa L. BTH-Induced MAP Kinase 2) plays a role in disease resistance responses. Overexpression of Rice OsBIMK2-overexpressing transgenic tobacco plants showed the enhanced disease resistance against both tomato mosaic virus and a fungal pathogen (Song et al., 2006).

Of these rice MAPKs involved in defense signaling, OsBWMK1 is most well identified so far. OsBWMK1 (Oryzae sativa L. Blast- and Wound - Induced MAP Kinase), which is activated by pathogen signals and localizes in the nucleus, carries a TDY phosphorylation motif instead of the more common TEY motif in its kinase domain and has an unusually extended C-terminal domain that is essential to its kinase activity and translocation to the nucleus (Cheong et al., 2003; He et al., 1999). The constitutive expression of OsBWMK1 in tobacco plants also enhances resistance against fungal and bacterial pathogen infections (Cheong et al., 2003). In addition, it was reported that OsBWMK1 phosphorylates both OsWRKY33 which binds to the Wbox element (TTGACCA) and OsEREBP1 that binds to the GCC box element (AGCCGCC) in several PR gene promoters, thereby enhancing DNA-binding activity of the factor to its in vitro cognate binding site. Transient coexpression of OsBWMK1 and OsWRKY33 or OsEREBP1 in Arabidopsis protoplast elevates SA-dependent expression of the GUS-reporter gene driven by the W-box element and the PR1 promoter for OsWRKY33 or by the GCC box element for OsEREBP1. Furthermore, the levels of SA and $\mathrm{H}_{2} \mathrm{O}_{2}$ are elevated in 35S-OsBWMK1 transgenic plants that show HR-like cell death. Altogether, OsBWMK1 may mediate plant defense responses by activating one or more transcription factors, such as OsWRKY33 or OsEREBP1 in plants (Cheong et al., 2003; Koo et al., 2009).

Recently, Reyna and Yang (2006) reported that nine of 17 OsMAPK genes were found to be induced at the mRNA level during either early, late, or both stages of infection upon inoculation with the blast fungus (Magnaporthe grisea), suggesting that about half of the rice MAPK genes are associated with pathogen infection and host defense response. Furthermore, it have been suggested that rice OsSIPK (rice ortholog of NtSIPK and AtMPK3) and its orthologs could acts as a central master switch for mediating plant responses against ozone, wounding and JA signals which known as defense-related signals in plant (Cho et al., 2009). However, there are no evidence on rice upstream kinase MAPKKK and MAPKK family involved in defense signal pathways so far.

\section{Functions of MAPK cascade pathways in plant ROS signaling}

Pathogen attacks trigger the production of reactive oxygen species (ROS) in plant and MAPK cascade pathways have been identified in oxidative stress mediated signaling (Apel and Hirt, 2004; Colcombet and Hirt, 2008). Tobacco SIPK and WIPK have been demonstrated to be activated by a variety of reactive oxygen species (Kumar and Klessig, 2000; Samuel et al., 2000). Interestingly, SIPK overexpressing transgenic tobacco showed the hypersensitive response to ozone which known to cause ROS accumulation in plants, suggesting tobacco SIPK acts as a MAPK regulator in ROS signaling (Samuel and Ellis, 2002). It have been reported that ozone also activates MPK3 and MPK6 in Arabidopsis (Ahlfors et al., 2004).

Yoshioka et al. (2003) reported that the MEK2 pathway in tobacco plants has been linked to ROS generation through the activation of $\mathrm{RBOH}$ (respiratory burst oxidase orthologue), which is known to produces ROS upon fungal infection. Furthermore, expression of constitutively active form of Arabidopsis MKK4 and MKK5 (orthologs of tobacco NtMEK2) enhanced generation of hydrogen peroxide and cell death (Ren et al., 2002). Arabidopsis MAPKKK MEKK1 has been also shown to mediate redox 


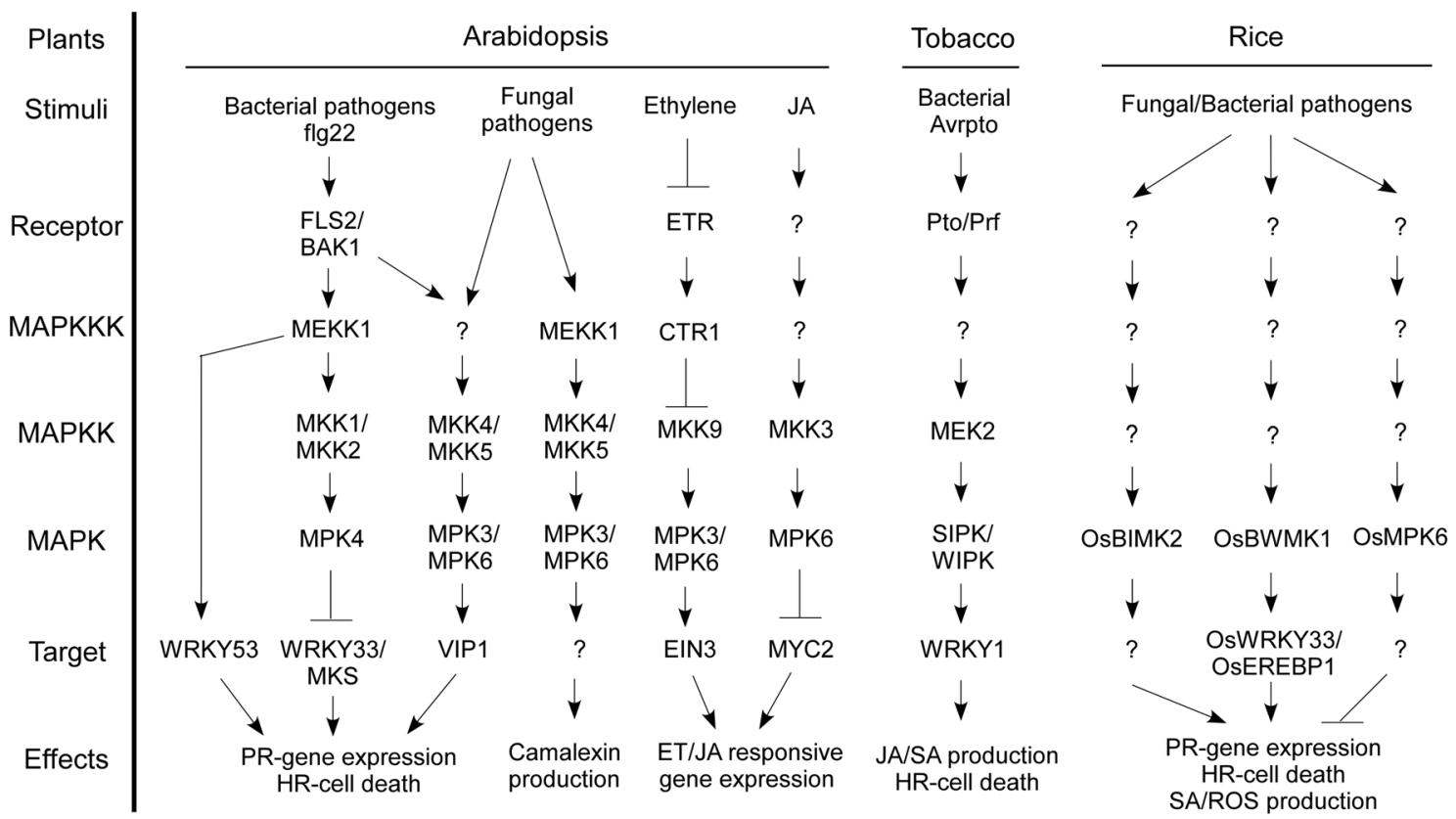

Fig. 1. Schematic illustration of plant MAPK cascades pathways involved in defense-related signal transduction from Arabidopsis, tobacco and rice. Plant defense signals-mediated MAPK cascades activate the several defense mechanisms, including induction of PRgene expression, cell wall deposition, hypersensitive response and phytoalexin (e.g. camalexin) synthesis. The scheme of general signal transduction pathway is shown on the left. "?" indicates unknown receptors and MAPK cascade components. PR; pathogen-responsive, ET; ethylene, JA; jasmonic acid, SA; salicylic acid, ROS; reactive oxygen species, HR; hypersensitive response, flg22; flagellin peptide elicitor.

homeostasis (Nakagami et al., 2006). MEKK1-deficient mutant plants (mekkl) showed the accumulation of ROSs and displayed aberrant regulation ROS-mediated genes. Interestingly, $m p k 4$ mutant plants showed similar phenotypes as mekkl mutant plants, and mekkl mutant lines were compromised in ROS-induced MPK4 activation, suggesting that MEKK1 may act as an upstream regulator of MPK4 in ROS signaling (Nakagami et al., 2006).

\section{Conclusions and Perspectives}

Plant MAPK cascades have been identified as a highly conserved signal molecules consisting of MAPKKKMAPKK-MAPK, which mediate plant cellular responses to biotic and abiotic stresses. It have also known that plant MAPK cascade modules play a important role in the regulation of defense responses by amplifying and transducing pathogen-related signals into altered gene expression (Fiil et al., 2009; Pitzschke et al., 2009). However, only a few effector-mediated components of MAPK cascades have been reported in plants (Fig. 1). Thus, further studies on identification of additional MAPK cascades modules and of downstream target molecules or pathways will help to determine the multiple networks of plant MAPK cascades pathways in defense signaling and may help in generating plant resistances into biotic and abiotic stresses in future.

\section{Acknowledgments}

This research was supported by a grant from the BioGreen 21 program (20080401034018), Rural Development Administration, Republic of Korea.

\section{References}

Agrawal, G K., Iwahashi, H. and Rakwal, R. 2003. Rice MAPKs. Biochem. Biophys. Res. Commun. 302: 171-180.

Ahlfors, R., Macioszek, V., Rudd, J., Brosche, M., Schlichting, R., Scheel, D. and Kangasjarvi, J. 2004. Stress hormone-independent activation and nuclear translocation of mitogen-activated protein kinases in Arabidopsis thaliana during ozone exposure. Plant J. 40:512-522.

Andreasson, E., Jenkins, T., Brodersen, P., Thorgrimsen, S., Petersen, N. H., Zhu, S., Qiu, J. L., Micheelsen, P., Rocher, A., Petersen, M., Newman, M. A., Bjorn-Nielsen, H., Hirt, H., Somssich, I., Mattsson, O. and Mundy, J. 2005. The MAP Kinase substrate MKS1 is a regulator of plant defense responses. EMBO J. 24:2579-2589.

Apel, K. and Hirt, H. 2004. Reactive oxygen species: metabolism, oxidative stress and signal transduction. Annu. Rev. Plant Biol. 
55:373-399

Asai, T., Tena, G, Plotnikova, J., Willmann, M. R., Chiu, W. L., Gomez-Gomez, L., Boller, T., Ausubel, F. M. and Sheen, J. 2002. MAP Kinase signaling cascade in Arabidopsis innate immunity. Nature 415:977-983.

Brodersen, P., Petersen, M., Bjorn-Nielsen, H., Zhu, S., Newman, M. A., Shokat, K. M., Rietz, S., Parker, J. and Mundy, J. 2006. Arabidopsis MAP kinase 4 regulates salicylic acid- and jasmonic acid/ethylene-dependent responses via EDS1 and PAD4. Plant J. 47:532-546.

Chang, L. and Karin, M. 2001. Mammalian MAP kinase signaling cascades. Nature 410:37-40.

Cheong, Y. H., Moon, B. C., Kim, J. K., Kim, C. Y., Kim, M. C., Kim, I. H., Park, C. Y., Kim, J. C., Park, B. O., Koo, S. C., Yoon, H. W., Chung, W. S., Lim, C. O., Lee, S. Y. and Cho, M. J. 2003. BWMK1, a rice mitogen-activated protein kinase, locates in the nucleus and mediates pathogenesis-related gene expression by activation of a transcription factor. Plant Physiol. 132:1961-1972.

Cho, K., Agrawal, G. K., Jwa, N. S., Kubo, A. and Rakwal, R. 2009. Rice OsSIPK and its orthologs: a central master switch for stress responses. Biochem. Biophys. Res. Commun. 379: 649-653.

Colcombet, J. and Hirt, H. 2008. Arabidopsis MAPKs: a complex signalling network involved in multiple biological processes. Biochem. J. 413:217-226.

Dangl, J. L. and Jones, J. D. 2001. Plant pathogens and integrated defense responses to infection. Nature 411:826-833.

Djamei, A., Pitzschke, A., Nakagami, H., Rajh, I. and Hirt, H. 2007. Trojan horse strategy in Agrobacterium transformation: abusing MAPK defense signaling. Science 318:453-456.

Fiil, B. K., Petersen, K., Petersen, M. and Mundy, J. 2009. Gene regulation by MAP kinase cascades. Curr. Opin. Plant Biol. 12:615-621.

Frye, C. A., Tang, D. and Innes, R. W. 2001. From the cover: negative regulation of defense responses in plants by a conserved MAPKK kinase. Proc. Natl. Acad. Sci. USA 98:373378.

Fu, S. F., Chou, W. C., Huang, D. D. and Huang, H. J. 2002. Transcriptional regulation of a rice mitogen-activated protein kinase gene, OsMAPK4, in response to environmental stress. Plant Cell Physiol. 43:958-963.

Hamel, L. P., Nicole, M. C., Sritubtim. S., Morency, M. J., Ellis, M., Ehlting, J., Beaudoin, N., Barbazuk, B., Klessig, D., Lee, J., Martin, G., Mundy, J., Ohashi. Y., Scheel, D., Sheen, J., Xing, T., Zhang, S., Seguin, A. and Ellis, B. E. 2006. Ancient signals: comparative genomics of plant MAPK and MAPKK gene families. Trends Plant Sci. 11:192-198.

Hanks, S. K., Quinn, A. M. and Hunter, T. 1988. The protein kinase family: conserved features and deduced phylogeny of the catalytic domains. Science 241:42-52.

He, C., Fong, S. H., Yang, D. and Wang, G. L. 1999. BWMK1, a novel MAP kinase induced by fungal infection and mechanical wounding in rice. Mol. Plant-Microbe Interact. 12:10641073.

Ichimura, K., Casais, C., Peck, S. C., Shinozaki, K. and Shirasu,
K. 2006. MEKK1 is required for MPK4 activation and regulates tissue-specific and temperature-dependent cell death in Arabidopsis. J. Biol. Chem. 281:36969-36976.

Ichimura, K., Mizoguchi, T., Yoshida, R., Yuasa, T. and Shinozaki, K. 2000. Various abiotic stresses rapidly activate Arabidopsis MAP kinases AtMPK4 and AtMPK6. Plant J. 24:655-665.

Jin, H., Liu, Y., Yang, K. Y., Kim, C. Y., Baker, B. and Zhang, S. 2003. Function of a mitogen-activated protein kinase pathway in $\mathrm{N}$ gene-mediated resistance in tobacco. Plant J. 33:719731.

Jonak, C., Okresz, L., Bogre, L. and Hirt, H. 2002. Complexity, cross talk and integration of plant MAP kinase signaling. Curr. Opin. Plant Biol. 5:415-424.

Kieber, J. J., Rothenberg, M., Roman, G., Feldmann, K. A. and Ecker, J. R. 1993. CTR1, a negative regulator of the ethylene response pathway in Arabidopsis, encodes a member of the raf family of protein kinase. Cell 72:427-441.

Kiegerl, S., Cardinale, F., Siligan, C., Gross, A., Baudouin, E., Liwosz, A., Eklof, S., Till, S., Bogre, L., Hirt, H. and Meskiene, I, 2000. SIMKK, a mitogen-activated protein kinase (MAPK) kinase, is a specific activator of the salt stress-induced MAPK, SIMK. Plant Cell 12:2247-2258.

Kim, C.Y. and Zhang, S. 2004. Activation of a mitogen-activated protein kinase cascade induces WRKY family of transcription factors and defense genes in tobacco. Plant J. 38:142-151.

Koo, S. C., Moo, B. C., Kim, J. K., Kim, C. Y., Sung, S. J., Kim, M. C., Cho, M. J. and Cheong, Y. H. 2009. OsBWMK1 mediates SA-dependent defense responses by activating the transcription factor OsWRKY33. Biochem. Biophys. Res. Commun. 387:365-370.

Kumar, D. and Klessig, D. F. 2000. Differential induction of tobacco MAP kinases by the defense signals nitric oxide, salicylic acid, ethylene, and jasmonic acid. Mol. Plant-Microbe Interact. 13:347-351.

Lieberherr, D., Thao, N. P., Nakashima, A., Umemura, K., Kawasaki, T. and Shimamoto, K. 2005. A sphingolipid elicitor-inducible mitogenactivated protein kinase is regulated by the small GTPase OsRac1 and heterotrimeric G-protein in rice. Plant Physiol. 138:1644-1652.

Liu, Y. and Zhang, S. 2004. Phosphorylation of 1-aminocyclopropane-1-carboxylic acid synthase by MPK6, a stress-responsive mitogen-activated protein kinase, induces ET biosynthesis in Arabidopsis. Plant Cell 16:3386-3399.

MAPK Group. 2002. Mitogen-activated protein kinase cascades in plants: a new nomenclature. Trends Plant Sci. 7:301-308.

Menke, F. L .H., Kang, H., Chen, Z., Park, J. M., Kumar, D. and Klessig, D. F. 2005. Tobacco transcription factor WRKY1 is phosphorylated by the MAP kinase SIPK and mediates HRlike cell death in tobacco. Mol. Plant-Microbe Interact. 18: 1027-1034.

Menke, F. L., van Pelt, J. A., Pieterse, C. M. and Klessig, D. F. 2004. Silencing of the mitogen-activated protein kinase MPK6 compromises disease resistance in Arabidopsis. Plant Cell 16: 897-907.

Meszaros, T., Helfer, A., Hatzimasoura, E., Magyar, Z., Serazetdinova, L., Rios, G, Bardoczy, V., Teige, M., Koncz, C., Peck, S. and 
Bogre, L. 2006. The Arabidopsis MAP kinase kinase MKK1 participates in defence responses to the bacterial elicitor flagellin. Plant J. 48:485-498.

Miao, Y., Laun, T. M., Smykowski, A. and Zentgraf, U. 2007. Arabidopsis MEKK1 can take a short cut: it can directly interact with senescence related WRKY53 transcription factor on the protein level and can bind to its promoter. Plant Mol. Biol. 65:63-76.

Mizoguchi, T., Irie, K., Hirayama, T., Hayashida, N., YamaguchiShinozaki, K., Matsumoto, K. and Shinozaki, K. 1996. A gene encoding a mitogen-activated protein kinase kinase kinase is induced simultaneously with genes for a mitogen-activated protein kinase and an S6 ribosomal protein kinase by touch, cold, and water stress in Arabidopsis thaliana. Proc. Natl. Acad. Sci. USA 93:765-769.

Nakagami, H., Kiegerl, S. and Hirt, H. 2004. OMTK1, a novel MAPKKK, channels oxidative stress signaling through direct MAPK interaction. J. Biol. Chem. 279:26959-26966.

Nakagami, H., Soukupova, H., Schikora, A., Zarsky, V. and Hirt, H. 2006. A Mitogen-activated protein kinase kinase kinase mediates reactive oxygen species homeostasis in Arabidopsis. J. Biol. Chem. 281:38697-38704.

Nishihama, R., Ishikawa, M., Araki, S., Soyano, T., Asada, T. and Machida, Y. 2001. The NPK1 mitogen-activated protein kinase kinase kinase is a regulator of cell-plate formation in plant cytokinesis. Genes Dev. 15:352-363.

Petersen, M., Brodersen, P., Naested, H., Andreasson, E., Lindhart, U., Johansen, B., Nielsen, H. B., Lacy, M., Austin, M. J., Parker, J. E., Sharma, S. B., Klessig, D. F., Martienssen, R., Mattsson, O., Jensen, A. B. and Mundy, J. 2000. Arabidopsis MAP kinase 4 negatively regulates systemic acquired resistance. Cell 103:1111-1120.

Pitzschke, A., Schikora, A. and Hirt, H. 2009. MAPK cascade signalling networks in plant defense. Curr. Opin. Plant Biol. $12: 421-426$.

Qiu, J. L., Fiil, B. K., Petersen, K., Nielsen, H. B., Botanga, C. J., Thorgrimsen, S., Palma, K., Suarez-Rodriguez, M. C., Sandbech-Clausen, S., Lichota, J., Brodersen, P., Grasser, K. D., Mattsson, O., Glazebrook, J., Mundy, J. and Petersen, M. 2008. Arabidopsis MAP kinase 4 regulates gene expression through transcription factor release in the nucleus. EMBO J. 27:2214-2221.

Quimby, B. B., Wilson, C. A. and Corbett, A. H. 2000. The interaction between Ran and NTF2 is required for cell cycle progression. Mol. Biol. Cell 11:2617-2629.

Ren, D., Liu, Y., Yang, K. Y., Han, L., Mao, G, Glazebrook, J. and Zhang, S. 2008. A fungal-responsive MAPK cascade regulates phytoalexin biosynthesis in Arabidopsis. Proc. Natl. Acad. Sci. USA 105:5638-5643.

Ren, D., Yang, H. and Zhang, S. 2002. Cell death mediated by MAPK is associated with hydrogen peroxide production in Arabidopsis. J. Biol. Chem. 277:559-565.

Reyna, N. S. and Yang, Y. 2006. Molecular analysis of the rice MAP kinase gene family in relation to Magnaporthe grisea infection. Mol. Plant-Microbe. Interact. 19:530-540.

Samuel, M. A. and Ellis, B. E. 2002. Double jeopardy: both overexpression and suppression of a redox-activated plant mitogen-activated protein kinase render tobacco plants ozone sensitive. Plant Cell 14:2059-2069.

Samuel, M. A., Miles. G. P. and Ellis. B. E. 2000. Ozone treatment rapidly activates MAP kinase signalling in plants. Plant J. 22: 367-376.

Schaffer, R., Landgraf, J., Accerbi, M., Simon, V., Larson, M. and Wisman, E. 2001. Microarray analysis of diurnal and circadian-regulated genes in Arabidopsis. Plant Cell 13:113-123.

Schoenbeck, M. A., Samac, D. A., Fedorova, M., Gregerson, R. G, Gantt, J. S. and Vance, C. P. 1999. The alfalfa (Medicago sativa) TDY1 gene encodes a mitogen-activated protein kinase homolog. Mol. Plant-Microbe Interact. 12:882-893.

Seo, S., Okamoto, M., Seto, H., Ishizuka, K., Sano, H. and Ohashi, Y. 1995. Tobacco MAP kinase: a possible mediator in wound signal transduction pathways. Science 270:1988-1992.

Song, D., Chen, J., Song, F. and Zheng, Z. 2006. A novel rice MAPK gene, OsBIMK2, is involved in disease-resistance responses. Plant Biol. 8:587-596.

Suarez-Rodriguez, M. C., Adams-Phillips, L., Liu, Y., Wang, H., Su, S. H., Jester, P. J., Zhang, S., Bent, A. F. and Krysan, P. J. 2007. MEKK1 is required for flg22-induced MPK4 activation in Arabidopsis plants. Plant Physiol. 143:661-669.

Takahashi, Y., Uehara, Y., Berberich, T., Ito, A., Saitoh, H., Miyazaki, A., Terauchi, R. and Kusano, T. 2004. A subset of hypersensitive response marker genes, including HSR203J, is the downstream target of a spermine signal transduction pathway in tobacco. Plant J. 40:586-595.

Tang, D. and Innes, R. W. 2002. Overexpression of a kinasedeficient form of the EDR1 gene enhances powdery mildew resistance and ethylene-induced senescence in Arabidopsis. Plant J. 32:975-983.

Teige, M., Scheikl, E., Eulgem, T., Doczi, R., Ichimura, K., Shinozaki, K., Dangl, J. L. and Hirt, H. 2004. The MKK2 pathway mediates cold and salt stress signaling in Arabidopsis. Mol. Cell 15:141-152.

Xiong, L. and Yang, Y. 2003. Disease resistance and abiotic stress tolerance in rice are inversely modulated by an abscisic acidinducible mitogen-activated protein kinase. Plant Cell 15:745759.

Xu. J., Li, Y., Wang, Y., Liu, H., Lei, L., Yang, H., Liu, G and Ren, D. 2008. Activation of MAPK kinase 9 induces ethylene and camalexin biosynthesis and enhances sensitivity to salt stress in Arabidopsis. J. Biol. Chem. 283:26996-27006.

Yang, K. Y., Liu, Y. and Zhang, S. 2001. Activation of a mitogenactivated protein kinase pathway is involved in disease resistance in tobacco. Proc. Natl. Acad. Sci. USA 98:741-746.

Yap, Y., Kodama, Y., Waller, F., Chung, K. M., Ueda, H., Nakamura, K., Oldsen, M., Yoda, H., Yamaguchi, Y. and Sano, H. 2005. Activation of a novel transcription factor through phosphorylation by WIPK, a wound-induced mitogen-activated protein kinase in tobacco plants. Plant Physiol. 139:127-137.

Yoshioka, H., Numata, N., Nakajima, K., Katou, S., Kawakita, K., Rowland, O., Jones, J. D. G and Doke, N. 2003. Nicotiana benthamiana $\mathrm{gp}^{91 \mathrm{phox}}$ homologs NbrbohA and NbrbohB participate in $\mathrm{H}_{2} \mathrm{O}_{2}$ accumulation and resistance to Phytophthora 
infestans. Plant Cell 15:706-718.

Yuan, B., Shen, X., Li, X., Xu, C. and Wang, S. 2007. Mitogenactivated protein kinase OsMPK6 negatively regulates rice disease resistance to bacterial pathogens. Planta 226:953-960.

Zhang, S. and Klessig, D. F. 1997. Salicylic acid activates a 48-kD MAP kinase in tobacco. Plant Cell 9:809-824.

Zhang, S. and Klessig, D. F. 1998. Resistance gene N-mediated de novo synthesis and activation of a tobacco mitogen-activated protein kinase by tobacco mosaic virus infection. Proc. Natl. Acad. Sci. USA 95:7433-7438.

Zhang, S. and Klessig, D. F. 2001. MAPK cascades in plant de- fense signaling. Trends Plant Sci. 6:520-527.

Zhang, S. and Liu, Y. 2001. Activation of salicylic acid-induced protein kinase, a mitogen-activated protein kinase, induces multiple defense responses in tobacco. Plant Cell 13:18771889.

Zhang, S., Liu, Y. and Klessig, D. F. 2000. Multiple levels of tobacco WIPK activation during the induction of cell death by fungal elicitins. Plant J. 23:339-347.

Zwerger, K. and Hirt, H. 2001. Recent advances in plant MAP kinase signalling. Biol. Chem. 382:1123-1131. 